\title{
The Oncologist's Guide to Synoptic Reporting: A Primer
}

\author{
Ekkehard Hewer \\ Institute of Pathology, University of Bern, Bern, Switzerland
}

\section{Keywords}

Synoptic reporting $\cdot$ Structured reporting $\cdot$ International Collaboration on Cancer Reporting

\begin{abstract}
Synoptic reporting in tumour pathology is defined by (1) completeness in terms of data elements as well as (2) a specific, laboratory value-like format. Adoption of synoptic reporting leads to more complete reporting of essential parameters, improved standardisation of diagnostic criteria and terminology, as well as easier retrieval of information. It is therefore associated with a high degree of satisfaction among end users including surgeons and oncologists and contributes to improvement of clinical care. Furthermore, synoptic reporting is an important step towards higher levels of data capture, which facilitate data exchange and analysis for quality assurance, cancer epidemiology and clinical and basic research. Increased interest in and adoption of synoptic reporting on a global level is stimulated by the International Collaboration on Cancer Reporting (ICCR) which publishes freely available, evidence-based datasets for reporting an increasing number of different cancer types. These developments pave the path for increased future application of synoptic reporting across the entire field of oncologic medicine, where it will likely deploy similar benefits to those in pathology. Given that synoptic reporting can be
\end{abstract}

karger@karger.com

(C) 2019 S. Karger AG, Basel

www.karger.com/ocl

Karger! considered the most precise means available for reporting of medical findings, it may be predicted to be critical for the promise of precision medicine to become real.

(c) 2019 S. Karger AG, Basel

\section{The Need for Complete and Standardised Reporting in Oncologic Pathology}

Oncologic pathology reports have a key role in diagnostic work-up, therapeutic management and post-therapeutic follow-up of every cancer patient. Given the multidisciplinarity of current oncologic management, it is natural that various specialists rely on different types of information. These specialists include, but are not limited to, medical and radiation oncologists, surgeons, diagnostic and interventional radiologists, nuclear medicine physicians and pathologists themselves. Additional stakeholders include cancer registries, clinical researchers, biobanking experts and quality managers. Furthermore, it is increasingly acknowledged that patients demand access to their reports - which in turn may influence how the information therein should be presented [1].

ORCID ID: 0000-0002-9128-0364
Ekkehard Hewer

Institute of Pathology, University of Bern

Murtenstrasse 31

CH-3008 Bern (Switzerland)

E-Mail ekkehard.hewer@ pathology.unibe.ch 
Table 1. Examples of information that might be found in laboratory or pathology reports in narrative and synoptic format, respectively

\begin{tabular}{|c|c|}
\hline Narrative & Synoptic \\
\hline $\begin{array}{l}\text { Upon incubation with the patient's serum, } \\
\text { immunofluorescence microscopy shows staining } \\
\text { of neutrophils in a perinuclear pattern; this is still } \\
\text { visible when the serum is diluted 1:320, but not } \\
\text { at 1:640 dilution }\end{array}$ & $\begin{array}{c}\text { ANCA: positive (p-ANCA); } \\
\text { Titer } 1: 320\end{array}$ \\
\hline \multicolumn{2}{|c|}{$\begin{array}{l}\text { The synoptic format of data element, paired with a response, recapitulates the way clinical laboratory values } \\
\text { are reported; this example of immunofluorescence illustrates that it would appear unusual to physicians to } \\
\text { receive such test results in narrative text, even though there may be a similar type of analysis underlying the } \\
\text { test result }\end{array}$} \\
\hline $\begin{array}{l}\ldots \text { with lymph node metastases detected in } 3 \text { out } \\
\text { of } 12 \text { lymph nodes, largest diameter } 1.2 \mathrm{~cm} \text {, } \\
\text { without evidence of extracapsular extension } \ldots\end{array}$ & $\begin{array}{l}\text { Number of lymph nodes submitted: } 12 \\
\text { Number of lymph nodes involved: } 3 \\
\text { Largest diameter of lymph node metastasis: } 1.2 \mathrm{~cm} \\
\text { Extranodal extension: not identified }\end{array}$ \\
\hline \multicolumn{2}{|c|}{$\begin{array}{l}\text { Example of typical elements from a surgical pathology report; most readers would likely find it easier to extrac } \\
\text { a particular piece of information from the synoptic as compared to the narrative report }\end{array}$} \\
\hline $\begin{array}{l}15 \% \text { of tumour cells are positive for Mum-1 } \\
15 \% \text { of tumour cells are positive for p53 } \\
15 \% \text { of tumour cells are positive for Ki-67 }\end{array}$ & $\begin{array}{l}\text { Mum-1 (immunostaining): negative } \\
\text { p53 (immunostaining): wild-type pattern } \\
\text { Ki-67 proliferation index: } 15 \%\end{array}$ \\
\hline \multicolumn{2}{|c|}{$\begin{array}{l}\text { In particular for biomarkers, specific criteria may have to be applied for interpretation of a given finding; } 15 \% \\
\text { of stained tumour cell nuclei would not qualify for Mum-1 expression in the context of the Hans Algorithm for } \\
\text { determining cell of origin in diffuse large B cell lymphoma; depending on how the immunostaining is set up, } \\
15 \% \text { of nuclear p53 staining would likely indicate wild-type TP53; in contrast, for Ki-67 the percentage of } \\
\text { positive nuclei is reported (with specific recommendations on how many nuclei to count for some tumour } \\
\text { types) }\end{array}$} \\
\hline
\end{tabular}

It would require almost supranatural abilities from a pathologist to keep all these stakeholders in mind when signing out reports and to address their needs - or even to know what all of these actually are in the context of each specific cancer type, histological subtype, type of specimen, tumour stage, eligibility for (neo-)adjuvant therapies, etc.

An additional level of complexity arises from the fact that is insufficient for a pathologist just to describe what they see under the microscope: the cut-off for a biomarker to be reported as positive or negative may vary depending on the context. For different organs there may be subtle differences in the diagnostic criteria for vascular invasion or in the definition of involvement of surgical margins. Furthermore, these classifications change over time - or there may be competing classifications or definitions at a given time point. Therefore, even a report given by the hypothetical near-supranatural pathologist mentioned above might lead to confusion, when it remains unclear what the underlying classifications and criteria were.

The Oncologist's Guide to Synoptic Reporting

\section{Synoptic Reporting}

Pathologists have long acknowledged these challenges and recognised Synoptic Reporting (derived from ancient Greek "syn-opsis" - overview) as a means to address them [2]. It was realised early on that the decision process of which parameters to include was critical and non-trivial $[3,4]$. Among a number of institutions, which have published protocols for synoptic reports in the past, two main players have emerged in recent years. The College of American Pathologists (CAP) publishes the most comprehensive set of synoptic cancer protocols as of now [5]. Their use has been mandatory for CAP-accredited laboratories which has been a major driver for synoptic reporting in the USA and internationally. More recently, an International Collaboration on Cancer Reporting (ICCR), sponsored by a variety of international pathology organisations, has been launched [6-8]. The ICCR has started to publish sets of protocols for various cancer types with the aim of covering the major cancer types. 
Both CAP and ICCR follow a strictly defined process for dataset development and consultation to ensure a broad consensus, expertise and reflection of the best evidence available.

\section{Format of Synoptic Reporting}

Initially, the term "synoptic" simply meant to indicate any structured format other than running text, usually with different data elements mentioned in separate lines [2]. CAP defines synoptic reports more narrowly [5], in that they must not only encompass a set of required data elements (RDE), but also adhere to a "paired format," where the designation of each RDE is followed by a "response." In essence, this is the way clinical laboratory values are reported (Table 1). Separate RDE must be displayed on separate lines.

Apart from this, CAP accepts a broad variety of possible formats and text markups. Of note, the range of acceptable formats includes low-technology implementations such as filling in and printing the protocols in $\mathrm{Mi}$ crosoft Word format or even photocopying protocols in order to fill them in manually. Similarly, the ICCR provides its protocols in $\mathrm{PDF}$, which can be printed and filled in manually. CAP specifically permits to present the RDE in any order and to include additional data elements at each institution's and/or pathologist's discretion [5]. Furthermore, additional narrative sections are acceptable.

\section{Terminology: Safety Issues and Uniformity}

Neither CAP nor ICCR have published detailed information on how specific wordings are chosen for data elements or responses. There are a number of recurrent themes, however: when comparing the various protocols and their development over time there is a strong tendency toward uniformity within and across protocols. Positive findings, for example, are usually reported as "present" rather than "yes" or "positive." Similarly, CAP protocols uniformly use the term "extranodal extension" rather than "extracapsular extension."

Negative findings are usually reported as "not identified" rather than "absent" along the line of the statement that "absence of evidence is not evidence of absence" and acknowledging the insight that in medicine the latter can rarely be provided. Of note, biomarkers for which the positive result reflects the normal situation are reported, for example, as "Intact nuclear expression" versus "Loss of nuclear expression" rather than "positive"/"negative."

Different responses to one data element are usually designed not to differ only by a single word, which might be accidentally omitted and thereby invert the intended meaning, e.g., "not identified" rather than "not present." Also, there is a tendency towards some degree of redundancy, such as in the case of grading, i.e., "G2, moderately differentiated" rather than just "G2."

All of the above conventions aim at minimisation of risks associated with misinterpretations of reports. Such considerations should be kept in mind while implementing synoptic protocols locally or translating them into different languages.

\section{Advantages of Synoptic Reporting}

A major advantage of synoptic over narrative reporting is an increase in completeness of data elements, as demonstrated by a number of studies across various cancer types, including - but not limited to - colorectal, lung, breast and prostate cancer, as well as cutaneous malignant melanoma [9-16]. One study of cutaneous malignant melanoma found completeness of reports to increase not only in a non-specialised, but also in a specialised setting [11]. A meta-analysis on the effects of synoptic reporting [16] found an increase in completeness in 13 out of 14 studies. This increase in completeness is critical, as lack of core data elements may affect the quality of cancer care $[10,17]$. Of note, the actual rates of completeness achieved by synoptic reporting vary significantly between studies, indicating that the characteristics of implementation may be an important factor. Furthermore, synoptic reporting may contribute to increased awareness of quality indicators and thereby improve the quality of pathologic evaluation. Interestingly, the metaanalysis mentioned above [16] found an increase in numbers of lymph nodes obtained from colorectal cancer resections as well as a higher percentage of specimens reaching the minimum of 12 lymph nodes upon introduction of synoptic reporting.

Overall, synoptic reporting is associated with a high degree of satisfaction among pathologists, surgeons and oncologists $[18,19]$. This satisfaction seems to be associated with perceived completeness of reports for the purpose of clinical decision making as well as ease of finding relevant information [18] (Fig. 1). 


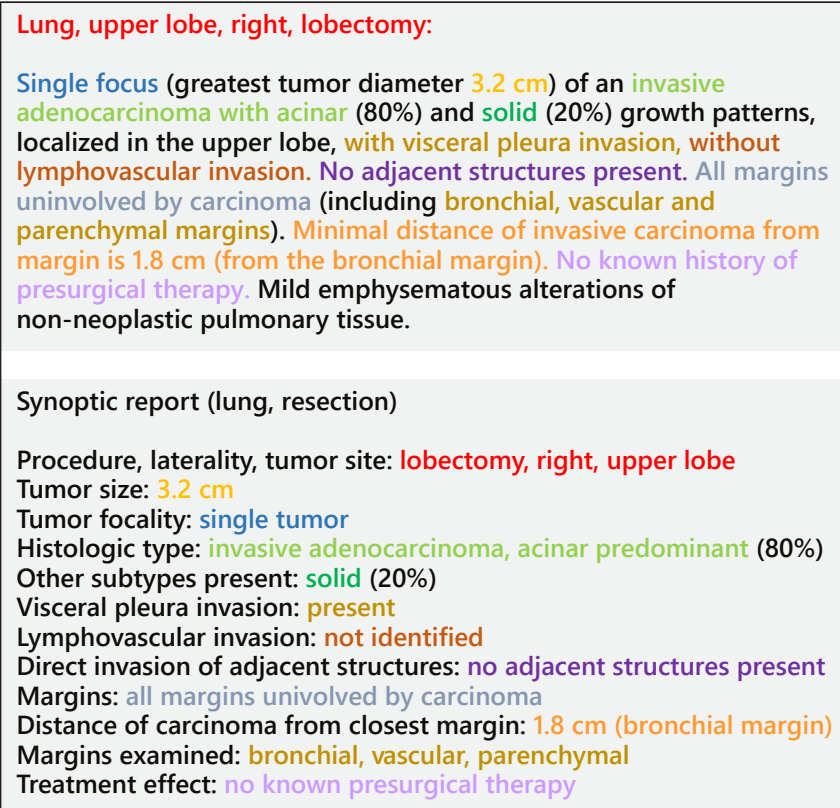

Fig. 1. Colour-coded representation of data elements (according to the College of American Pathologists template for lung cancer) in narrative (top) and synoptic (bottom) formats. Even when complete in terms of required data elements, narrative reports tend to be shorter than synoptic reports. Finding a particular piece of information, however, is easier with the synoptic format. As in this example, narrative reports tend to include more non-essential data of little clinical relevance (mild emphysematous change) than synoptic reports, while essential data elements are often incomplete.

\section{Limitations of Synoptic Reporting}

The overall high level of satisfaction with synoptic reporting notwithstanding, a similarly recurrent theme across various studies is that pathologists need more time to complete synoptic as compared to narrative reports [18]. Generally, however, the increment in time was moderate and found acceptable when considering the benefits of synoptic reporting.

An additional issue may be the length of reports. Most of the CAP protocol files extend over several pages. ICCR templates are more compact but may be challenging to render within an existing laboratory information system because of their complex formatting with two columns. In part, increased length of reports is an intrinsic consequence of completeness in terms of RDE as well as of the synoptic format itself. Nevertheless, overly long reports can be avoided by a number of means. First, many RDE are conditional, i.e., they may be mandatory only in a subset of cases (e.g., nuclear grading does not apply to chro-

The Oncologist's Guide to Synoptic

Reporting mophobe renal cell carcinoma). In that case, it is acceptable to omit the pertinent line completely, rather than reporting the RDE as "not applicable." Second, most CAP and ICCR protocols contain a number of optional data elements, which may or may not be reported at each pathologist's or institution's discretion. It may be prudent in this context to refrain from including "everything," but rather to keep readability of reports in mind. Along the same line, some hesitance may be advisable with regard to including additional data elements on a local basis.

Finally, synoptic protocols may not fit very specific circumstances well, such as two different histologic tumour types (e.g., carcinoma and lymphoma) occurring in the same resection specimens. Usually, however, such issues can be addressed in a satisfactory manner, and the possibility to include free text provides sufficient flexibility.

\section{How to Read Synoptic Reports}

In most instances, synoptic reports should be sufficiently self-explanatory in order to be well understandable to physicians with at least some understanding of the respective medical field. In particular, preferences of individual pathologists with respect to wording should be less of an issue than with narrative reports. Furthermore, as cancer protocols are continuously updated, synoptic reports will usually contain the information required for patient management in current terminology and with sufficient granularity. When very specific information is required, the notes accompanying each cancer or biomarker protocol may be a useful resource. CAP protocols contain a "Notes" section, which gives very detailed information on diagnostic criteria, cut-offs, grading schemes, etc. ICCR protocols are available in bookmarked and hyperlinked versions containing similar information. The respective documents are freely available on the CAP and ICCR websites. Ideally, a synoptic report should contain information as to which version of which protocol it refers. This is of particular relevance with regard to future users, given that classifications change over time.

\section{Synoptic Reporting on the Path Towards Higher Levels of Data Capture}

As discussed by Ellis and Srigley [20, 21], synoptic reporting has a middle position regarding the degree to which data is structured and is classified as Level 3 in a 6-tiered system: 
- Level 1: Narrative report (no defined content)

- Level 2: Narrative report with standardised content (e.g., by using a checklist for dictation)

- Level 3: Synoptic report - adds a specific format, but not necessarily any underlying software implementation

- Level 4: Synoptic report with electronic reporting tools

- Level 5: Standardised structured report with underlying database structure

- Level 6: Standardised structured report with binding terminology in order to facilitate data exchange

According to Ellis and Srigley, implementation of Level 3 primarily benefits immediate clinical needs, while higher levels of data capture are necessary for synoptic reporting to unfold its full potential for pathologists and secondary users. An underlying database structure will allow pathologists to easily monitor statistical distribution of findings and thereby identify potential deviations from expected frequencies, which in turn might point towards issues on a technical or interpretational level.

Ultimately, linking synoptic reports and databases with a uniform terminology, such as SNOMED-CT, will allow third parties, including biobanks and cancer registries, to access large datasets with unprecedented granularity.

Nevertheless, synoptic reporting according to Level 3 in the Ellison/Srigley classification has an important role within the path towards higher levels of data capture: it already serves one particular purpose, i.e., clinicians' needs, very well. Furthermore, it is usually feasible with standard features of pathology information systems so that it can be implemented more easily, faster and without major financial implications. Finally, it may be a very significant step on a psychological level, as it trains users to adhere to a standardised format and terminology and fosters precision in reporting.

\section{Synoptic Reporting in Oncology beyond Pathology}

While the historic origin and widest application of synoptic reporting are in oncologic pathology, its concepts are spreading to non-neoplastic pathology $[22,23]$ as well as oncologic specialities other than pathology. Main areas of application of synoptic reporting include radiology [24-28] and operative reports in surgery [29-34]. While the overall number of studies addressing the effects of synoptic reporting is considerably lower than in pathology, they tend to show similar outcomes; completeness of reports increases with the use of synoptic reporting, while at the same time the amount of non-essential information is reduced [35-37]. A web-based synoptic reporting tool for thyroid surgery was found to achieve $100 \%$ completeness of essential prognostic factors while completeness varied between 3 and $>95 \%$ for various parameters in descriptive operative reports [38]. Of note, initiatives for synoptic reporting in radiology or oncologic surgery are mostly driven by single academic centres. In contrast to pathology, so far there is only a limited role of national or international professional or scientific societies. One exception is the American Thyroid Association, which has issued a statement regarding essential elements of perioperative information in relation to thyroid surgery and endorsed the use of synoptic operative reports [32].

\section{Perspective}

Over more than a quarter of a century, the concept of synoptic reporting in pathology has matured from local initiatives [2] to international standardisation with defined processes for design and maintenance of evidencebased reporting templates which are coordinated with the World Health Organization Classification of Tumours [6, $21,39]$. Data are increasingly structured and linked to ontologies such as SNOMED-CT and LOINC [40], facilitating unprecedented levels of integration with the potential to revolutionize their use with regard to clinical care, quality assurance, as well as clinical and basic research [41].

This development paves the path for future widespread applications of synoptic reporting (and higher levels of data capture) in other fields in oncology. Not only do the forces which have driven this development in pathology - the need for complete, accurate and standardised information - act on all oncologic specialties, but also synoptic reporting can be predicted to be a major part of the respective solutions. Experience from pathology shows that high quality, evidence-based and timely consensus forms for reporting and their endorsement by national and international professional and scientific societies are critically important facilitators for widespread application of synoptic reporting.

For precision medicine not to remain an empty promise, precision has to be the modus operandi in the entire practice of oncologic medicine, and synoptic reporting is the most precise type of communication available to us.

\section{Disclosure Statement}

The author has no conflicts of interest to declare. 


\section{References}

1 Lee CI, Langlotz CP, Elmore JG. Implications of Direct Patient Online Access to Radiology Reports Through Patient Web Portals. J Am Coll Radiol. 2016 Dec;13(12 12 Pt B):1608-14.

2 Markel SF, Hirsch SD. Synoptic surgical pathology reporting. Hum Pathol. 1991 Aug; 22(8):807-10.

3 Dworak O, Markei SF, Hirsch SD. Synoptic surgical pathology reporting. Hum Pathol. 1992 Jan;23(1):85-6.

4 Chamberlain DW, Wenckebach GF, Alexander F, Fraser RS, Kolin A, Newman T. Pathological examination and the reporting of lung cancer specimens. Clin Lung Cancer. 2000 May; 1(4):261-8.

5 College of American Pathologists. Cancer protocol templates [cited 2019 May 4]. Available from: www.cap.org.

6 International Collaboration on Cancer Reporting (ICCR). Cancer datasets [cited 2019 May 4]. Available from: www.iccr-cancer. org.

7 Bullock MJ, Beitler JJ, Carlson DL, Fonseca I, Hunt JL, Katabi N, Sloan P, Taylor SM, Williams MD, Thompson LDR: Data Set for the Reporting of Nodal Excisions and Neck Dissection Specimens for Head and Neck Tumors: Explanations and Recommendations of the Guidelines From the International Collaboration on Cancer Reporting (ICCR). Arch Pathol Lab Med. 2019 Apr; 143(4):452-62.

8 Nicholson AG, Detterbeck F, Marx A, Roden AC, Marchevsky AM, Mukai K, et al. Dataset for reporting of thymic epithelial tumours: recommendations from the International Collaboration on Cancer Reporting (ICCR). Histopathology. 2017 Mar;70(4): 522-38.

9 Messenger DE, McLeod RS, Kirsch R. What impact has the introduction of a synoptic report for rectal cancer had on reporting outcomes for specialist gastrointestinal and nongastrointestinal pathologists? Arch Pathol Lab Med. 2011 Nov;135(11):1471-5.

10 Haydu LE, Holt PE, Karim RZ, Madronio CM, Thompson JF, Armstrong BK, et al. Quality of histopathological reporting on melanoma and influence of use of a synoptic template. Histopathology. 2010 May;56(6): 768-74.

11 Karim RZ, van den Berg KS, Colman MH, McCarthy SW, Thompson JF, Scolyer RA. The advantage of using a synoptic pathology report format for cutaneous melanoma. Histopathology. 2008 Jan;52(2):130-8.

12 Aumann K, Amann D, Gumpp V, Hauschke D, Kayser G, May AM, et al. Template-based synoptic reports improve the quality of pathology reports of prostatectomy specimens. Histopathology. 2012 Mar;60(4):634-44.
13 Aumann K, Kayser G, Amann D, Bronsert P, Hauschke D, Palade E, et al. The format type has impact on the quality of pathology reports of oncological lung resection specimens. Lung Cancer. 2013 Sep;81(3):382-7.

14 Aumann K, Niermann K, Asberger J, Wellner U, Bronsert P, Erbes T, et al. Structured reporting ensures complete content and quick detection of essential data in pathology reports of oncological breast resection specimens. Breast Cancer Res Treat. 2016 Apr; 156(3):495-500.

15 Maley A, Patrawala S, Stoff B. Compliance with the College of American Pathologists Protocol for Melanoma in Synoptic and Non-Synoptic reports: A cross-sectional study. J Am Acad Dermatol. 2016 Jan;74(1): 179-81.

16 Sluijter CE, van Lonkhuijzen LR, van Slooten HJ, Nagtegaal ID, Overbeek LI. The effects of implementing synoptic pathology reporting in cancer diagnosis: a systematic review. Virchows Arch. 2016 Jun;468(6):639-49.

17 Verleye L, Ottevanger PB, Kristensen GB, Ehlen T, Johnson N, van der Burg ME, et al. Quality of pathology reports for advanced ovarian cancer: are we missing essential information? An audit of 479 pathology reports from the EORTC-GCG 55971/NCICCTG OV13 neoadjuvant trial. Eur J Cancer. 2011 Jan;47(1):57-64.

18 Lankshear S, Srigley J, McGowan T, Yurcan M, Sawka C. Standardized synoptic cancer pathology reports - so what and who cares? A population-based satisfaction survey of 970 pathologists, surgeons, and oncologists. Arch Pathol Lab Med. 2013 Nov;137(11): 1599-602.

19 Hammond EH, Flinner RL. Clinically relevant breast cancer reporting: using process measures to improve anatomic pathology reporting. Arch Pathol Lab Med. 1997 Nov; 121(11):1171-5.

20 Ellis DW. Surgical pathology reporting at the crossroads: beyond synoptic reporting. $\mathrm{Pa}$ thology. 2011 Aug;43(5):404-9.

21 Ellis DW, Srigley J. Does standardised structured reporting contribute to quality in diagnostic pathology? The importance of evidence-based datasets. Virchows Arch. 2016 Jan;468(1):51-9.

22 Benton SJ, Lafreniere AJ, Grynspan D, Bainbridge SA. A synoptic framework and future directions for placental pathology reporting. Placenta. 2019 Feb;77:46-57.

23 Edwards PC. Is it time to extend synoptic reporting to include potentially preneoplastic oral epithelial and lichenoid lesions? Oral Surg Oral Med Oral Pathol Oral Radiol. 2017 Aug;124(2):105-6.

24 Panicek DM, Hricak H. Recommendations for the Initial Cancer Staging MRI Report. J Magn Reson Imaging. 2019 May;49(5): 1209-11.
25 Patel A, Rockall A, Guthrie A, Gleeson F, Worthy S, Grubnic S, et al. Can the completeness of radiological cancer staging reports be improved using proforma reporting? A prospective multicentre non-blinded interventional study across 21 centres in the UK. BMJ Open. 2018 Oct;8(10):e018499.

26 Kennedy ED, Milot L, Fruitman M, Al-Sukhni E, Heine G, Schmocker S, et al. Development and implementation of a synoptic MRI report for preoperative staging of rectal cancer on a population-based level. Dis Colon Rectum. 2014 Jun;57(6):700-8.

27 Spiegle G, Leon-Carlyle M, Schmocker S, Fruitman M, Milot L, Gagliardi AR, et al. Development of a synoptic MRI report for primary rectal cancer. Implement Sci. 2009 Dec; 4(1):79.

28 Dhillon RS, King JA, Goldschlager T, Wang YY. Synoptic reporting of pituitary magnetic resonance imaging. ANZ J Surg. 2018 May; 88(5):460-3.

29 Ehrlich PF. Reply to: Synoptic operative reports for quality improvement in pediatric cancer care: Surgical protocol violations in children with renal tumors provides an opportunity to improve pediatric cancer care: A report from the Children's Oncology Group. Pediatr Blood Cancer. 2018 Oct; 65(10):e27277.

30 Cundy TP, Kirby CP, Kirby ML. Synoptic operative reports for quality improvement in pediatric cancer care. Pediatr Blood Cancer. 2018 Oct;65(10):e27238.

31 Brower ST, Katz M, Pisters P, Merchant N, Weber S, Posner M. Electronic synoptic operative reporting for pancreatic resection. J Am Coll Surg. 2011 Mar;212(3):425-6.

32 Carty SE, Doherty GM, Inabnet WB 3rd, Pasieka JL, Randolph GW, Shaha AR, et al.; Surgical Affairs Committee Of The American Thyroid Association. American Thyroid Association statement on the essential elements of interdisciplinary communication of perioperative information for patients undergoing thyroid cancer surgery. Thyroid. 2012 Apr;22(4):395-9.

33 Badruddoja M. Electronic synoptic operation report. J Am Coll Surg. 2011 Feb;212(2): 271-2.

34 Temple WJ, Chin-Lenn L, Mack LA; Cancer Surgery Alberta. Evaluating populationbased breast cancer surgical practice in real time with a web-based synoptic operative reporting system. Am J Surg. 2014 May;207(5): 693-6.

35 Eng JL, Baliski CR, McGahan C, Cai E. Completeness of breast cancer operative reports in a community care setting. Breast. 2017 Oct;35:91-7. 
36 King S, Dimech M, Johnstone S. Structured pathology reporting improves the macroscopic assessment of rectal tumour resection specimens. Pathology. 2016 Jun;48(4):34952.

37 Maniar RL, Hochman DJ, Wirtzfeld DA, McKay AM, Yaffe CS, Yip B, et al. Documentation of quality of care data for colon cancer surgery: comparison of synoptic and dictated operative reports. Ann Surg Oncol. 2014 Oct;21(11):3592-7.
38 Chambers AJ, Pasieka JL, Temple WJ. Improvement in the accuracy of reporting key prognostic and anatomic findings during thyroidectomy by using a novel Web-based synoptic operative reporting system. Surgery. 2009 Dec;146(6):1090-8.

39 Compérat E, Babjuk M, Algaba F, Amin M, Brimo F, Grignon D, et al. SIU-ICUD on bladder cancer: pathology. World J Urol. 2019 Jan;37(1):41-50.
40 Campbell WS, Karlsson D, Vreeman DJ, Lazenby AJ, Talmon GA, Campbell JR. A computable pathology report for precision medicine: extending an observables ontology unifying SNOMED CT and LOINC. J Am Med Inform Assoc. 2017 Sep.

41 Banz Y, Berezowska S, de Leval L, RubbiaBrandt L, Tolnay M, Moch H, et al. Advancing synoptic cancer reports beyond English: the University of Bern/PathoLink approach. Virchows Arch. 2018 Nov;473(5):655-6. 